\title{
TYPICAL CONTINUOUS FUNCTIONS ARE VIRTUALLY NONMONOTONE
}

\author{
P. HUMKE AND M. LACZKOVICH ${ }^{1}$
}

\begin{abstract}
For every porosity premeasure $\phi$, a typical continuous function meets every monotone function in a bilaterally strongly $\phi$-porous set. The statement does not remain valid if we replace the class of monotone functions by the class of absolutely continuous functions.
\end{abstract}

1. This paper continues a recent study of intersections of typical continuous functions with certain classes of functions. By the term "typical continuous function" we mean that the set of all functions which have the property under consideration is a residual subset of the complete metric space $C[0,1]$, and by the set in which two functions intersect we mean the set of domain values at which the two functions coincide. In [T], B. S. Thomson proved that a typical continuous function intersects every constant function in a bilaterally strongly porous set. A set $E \subset \mathbf{R}$ is bilaterally strongly porous if for every $x \in E$ there are sequences of intervals

$$
I_{n} \subset(x-1 / n, x) \backslash E \text { and } J_{n} \subset(x, x+1 / n) \backslash E
$$

such that

$$
\lim _{n \rightarrow \infty} \frac{\operatorname{dist}\left(x, I_{n}\right)}{\left|I_{n}\right|}=\lim _{n \rightarrow \infty} \frac{\operatorname{dist}\left(x, J_{n}\right)}{\left|J_{n}\right|}=0 .
$$

In [H], J. Haussermann generalizes Thomson's result in two ways, replacing the class of constant functions by a larger class and the notion of bilateral strong porosity by a finer notion. We call a continuous function $\phi:(0,1] \rightarrow(0,1]$ a porosity premeasure. A set $E \subset \mathbf{R}$ ins bilaterally strongly $\phi$-porous if, for every $x \in E$, there are sequences of intervals $I_{n}$ and $J_{n}$ as above such that

$$
\lim _{n \rightarrow \infty} \frac{\operatorname{dist}\left(x, I_{n}\right)}{\phi\left(\left|I_{n}\right|\right)}=\lim _{n \rightarrow \infty} \frac{\operatorname{dist}\left(x, J_{n}\right)}{\phi\left(\left|J_{n}\right|\right)}=0 .
$$

Haussermann proves that for each porosity premeasure $\phi$, a typical continuous function intersects every function with finite Dini derivates in a bilaterally strongly

Received by the editors July 2, 1984 .

1980 Mathematics Subject Classification. Primary 26A48, 26A15, 26A99.

Key words and phrases. Typical continuous functions, intersections with monotone functions, porous sets.

${ }^{1}$ This work was done while the authors participated in a Special Year in Real Analysis at the University of California, Santa Barbara. 
$\phi$-porous set. In this paper we prove, answering a question of D. R. Andy and J. Haussermann, that the same result is true if the class of functions with finite Dini derivates is replaced by the class of monotone functions (Theorem 1). On the other hand, A. M. Bruckner points out that the statement is not true for the class of functions of bounded variation (moreover, absolutely continuous functions). We present his argument in a simplified form in Theorem 3.

2. A pair of sequences $\alpha=\left\{\alpha_{n}\right\}$ and $\beta=\left\{\beta_{n}\right\}$ is called proper if $\left\{\beta_{n}\right\} \rightarrow 0$ and $0<\alpha_{n}<\beta_{n}$ for $n=1,2, \ldots$ If $(\alpha, \beta)$ is a proper pair of sequences, then a sequence $x=\left\{x_{n}\right\}$ is called an $(\alpha, \beta)$-sequence if $x$ converges (to, say, $x_{0}$ ) and $\alpha_{n} \leqslant x_{n}-x_{0}$ $\leqslant \beta_{n}$ for $n=1,2, \ldots$. Now if $(\alpha, \beta)$ is a fixed proper pair, define

$I_{n}=\left\{f \in C[0,1]:\right.$ there is an $(\alpha, \beta)$-sequence $x$ with $f$ increasing on $\left.\left\{x_{i}\right\}_{i=n}^{\infty}\right\}$, $D_{n}=\left\{f \in C[0,1]:\right.$ there is an $(\alpha, \beta)$-sequence $x$ with $f$ decreasing on $\left.\left\{x_{i}\right\}_{i=n}^{\infty}\right\}$.

LEMMA 1. If $(\alpha, \beta)$ is proper, then both $I_{n}$ and $D_{n}$ are closed for every $n=1,2, \ldots$

Proof. As $D_{n}=\left\{f:-f \in I_{n}\right\}$, it suffices to prove the result for $I_{n}, n=1,2, \ldots$ Let $N$ be fixed and suppose $\left\{f_{k}\right\}_{k=1}^{\infty} \subset I_{N}$ with $\left\{f_{k}\right\} \rightarrow f$ uniformly. As $f_{k} \in I_{N}$ for $k=1,2, \ldots$, it follows that for each $k$ there is a sequence $x^{k}=\left\{x_{n}^{k}\right\}_{n=1}^{\infty}$ converging to, say, $x_{0}^{k}$, such that $\alpha_{n} \leqslant x_{n}^{k}-x_{0}^{k} \leqslant \beta_{n}$ for every $n$, and $f_{k}$ is increasing on $\left\{x_{n}^{k}\right\}_{n=N}^{\infty}$ for each $k$. Using a familiar diagonal argument, one obtains a subsequence $\left\{k_{i}\right\}_{i=1}^{\infty}$ such that, for each $n=0,1,2, \ldots$, the corresponding sequence $\left\{x_{n}^{k_{i}}\right\}_{i=1}^{\infty}$ converges (to, say, $x_{n}^{*}$ ). As $\alpha_{n} \leqslant x_{n}^{k_{i}}-x_{0}^{k_{i}} \leqslant \beta_{n}$ it follows that $\alpha_{n} \leqslant x_{n}^{*}-x_{0}^{*} \leqslant \beta_{n}$ and, as such, $x^{*}=\left\{x_{n}^{*}\right\}$ is an $(\alpha, \beta)$-sequence. As $\left\{f_{k}\right\} \rightarrow f$ uniformly and each $f_{k}$ is increasing on $\left\{x_{n}^{k}\right\}_{n=N}^{\infty}$ it follows that $f$ is increasing on $\left\{x_{n}^{*}\right\}_{n=N}^{\infty}$ and, hence, $f \in I_{N}$.

LEMMA 2. If $(\alpha, \beta)$ is proper, then both $I_{n}$ and $D_{n}$ are nowhere dense for every $n=1,2, \ldots$

Proof. Again it suffices to prove the result for $I_{n}, n=1,2, \ldots$, and in light of Lemma 1 we need only show $I_{n}$ contains no ball. Let $N$ be fixed, $f \in C[0,1]$, and $\varepsilon>0$. We must find a $g \in C[0,1] \backslash I_{N}$ within $\varepsilon$ of $f$.

First, partition $[0,1]$ into congruent closed intervals $\left\{J_{k}: k=1,2, \ldots, K\right\}$ each of length $d<\varepsilon / 2$ such that the oscillation of $f$ on each of them is less than $\varepsilon / 2$. Let $n_{1}, n_{2} \geqslant N$ be such that

$$
0<\alpha_{n_{2}}<\beta_{n_{2}}<\alpha_{n_{1}}<\beta_{n_{1}}<d
$$

and let $\delta=\min \left\{\alpha_{n_{2}},\left(d-\beta_{n_{1}}\right) / 2\right\}$. We define $g$ on each interval $I_{k}$ separately. Let $k$ be fixed and suppose $J_{k}=[a, b]$.

1. If $f(a)>f(b)$, define $g$ to be linear on $J_{k}$ and to agree with $f$ at $a$ and $b$.

2. If $f(a) \leqslant f(b)$, define $g$ in such a way that $g$ is linear on each of $[a, a+\delta]$ and $[a+\delta, b]$, has slope -1 on $[a+\delta, b]$, and agrees with $f$ at $a$ and $b$.

As the oscillation of $f$ on each $J_{k}$ is less than $\varepsilon / 2$, it follows that $|f(x)-g(x)|<\varepsilon$ for each $x \in[0,1]$. Now, let $\left\{x_{n}\right\}$ be an $(\alpha, \beta)$-sequence which converges to $x_{0} \in[0,1)$. Then there is a unique $k$ such that $x_{0} \in J_{k}$ but $x_{0}$ is not the right 
endpoint of $J_{k}$. We again denote $J_{k}$ by $[a, b]$. If $f(a)>f(b)$, then $g$ is decreasing on $J_{k}$ and hence eventually decreasing on $\left\{x_{n}\right\}$. Suppose now, that $f(a) \leqslant f(b)$. If $x_{0} \in[a+\delta, b)$, then again $g$ is decreasing on $[a+\delta, b]$ and eventually on $\left\{x_{n}\right\}$. Hence, we may suppose $x_{0} \in[a, a+\delta)$, and we obtain

$$
\begin{aligned}
a+\delta & \leqslant a+\alpha_{n_{2}} \leqslant x_{0}+\alpha_{n_{2}} \leqslant x_{n_{2}} \leqslant x_{0}+\beta_{n_{2}}<x_{0}+\alpha_{n_{1}} \\
& \leqslant x_{n_{1}} \leqslant x_{0}+\beta_{n_{1}}<a+\delta+\beta_{n_{1}}<b .
\end{aligned}
$$

However, $g$ is decreasing on $[a+\delta, b]$ and so $g\left(x_{n_{2}}\right)>g\left(x_{n_{1}}\right)$; that is, $g$ is not increasing on $\left\{x_{n}\right\}_{n=N}^{\infty}$ and as this sequence was arbitrary, the lemma is established.

THEOREM 1. Let $\phi$ be an arbitrary porosity premeasure. Then a typical continuous function intersects every monotone function in a bilaterally strongly $\phi$-porous set.

Proof. First we define a proper pair of sequences $(\alpha, \beta)$ as follows. Let $\beta_{0}=1$ and, if $\beta_{n} \in(0,1]$ has been defined, define $0<\beta_{n+1}<\min \left(\beta_{n}, 1 /(n+1)\right)$ such that $\phi\left(\beta_{n}-\beta_{n+1}\right)>n \beta_{n+1}$. Let $\alpha_{n}=\beta_{n+1}, n=0,1,2, \ldots$ It follows from Lemmas 1 and 2 that if $f$ is a typical continuous function on $[0,1]$ and $f$ is monotone on a set $M \subset[0,1]$, then for every $x \in M$

$$
M \cap\left[x+\alpha_{n_{i}}, x+\beta_{n_{i}}\right]=\varnothing
$$

for a subsequence $n_{i}$ of natural numbers. Let $J_{i}=\left[x+\alpha_{n_{i}}, x+\beta_{n_{i}}\right], i=1,2, \ldots$ Then $J_{i} \subset(x, x+1 / i) \backslash M$ and

$$
\operatorname{dist}\left(x, J_{i}\right)=\alpha_{n_{i}}=\beta_{n_{i}+1}<\frac{\phi\left(\beta_{n_{i}}-\beta_{n_{i}+1}\right)}{n_{i}}=\frac{\phi\left(\left|J_{i}\right|\right)}{n_{i}}, \quad i=1,2, \ldots
$$

Therefore,

$$
\lim _{i \rightarrow \infty} \frac{\operatorname{dist}\left(x, J_{i}\right)}{\phi\left(\left|J_{i}\right|\right)}=0 .
$$

Using a similar argument we can find a sequence $I_{i} \subset(x-1 / i, x) \backslash M$ with

$$
\lim _{i \rightarrow \infty} \frac{\operatorname{dist}\left(x, I_{i}\right)}{\phi\left(\left|I_{i}\right|\right)}=0
$$

which proves that $M$ is bilaterally strongly $\phi$-porous.

The following theorem is essentially due to J. Haussermann [H]. Since both the statement and proof become slightly simpler using our $(\alpha, \beta)$ notation, we provide the proof.

THEOREM 2. Let $(\alpha, \beta)$ be a proper pair of sequences and let $\sigma$ be a positive increasing function on $(0,1]$ with

$$
\lim _{n \rightarrow \infty} \frac{\sigma\left(\alpha_{n}\right) \beta_{n}}{\alpha_{n}}=\infty .
$$

If $f \in C[0,1]$ is arbitrary, then for a.e. $x \in[0,1]$ there is an $(\alpha, \beta)$-sequence $\left\{y_{n}\right\} \rightarrow x$ such that

$$
\left|f\left(y_{n}\right)-f(x)\right| \leqslant \sigma\left(y_{n}-x\right)
$$

for $n$ sufficiently large. 
Proof. Let $f \in C[0,1]$ and suppose $|f(x)| \leqslant K, x \in[0,1]$. Define

$$
\begin{aligned}
E^{+}=\{ & x \in[0,1): \text { for infinitely many } n, \\
& \left.f(y)-f(x)>\sigma(y-x) \text { whenever } y \in\left[x+\alpha_{n}, x+\beta_{n}\right]\right\}, \\
E^{-}=\{ & x \in[0,1) ; \text { for infinitely many } n, \\
& \left.f(y)-f(x)<-\sigma(y-x) \text { whenever } y \in\left[x+\alpha_{n}, x+\beta_{n}\right]\right\} .
\end{aligned}
$$

We have to show $\lambda\left(E^{+}\right)=\lambda\left(E^{-}\right)=0$. Let $\varepsilon>0$ be given. There is an $n_{0}$ such that $\beta_{n}<1$ and $\alpha_{n}<\varepsilon \sigma\left(\alpha_{n}\right) \beta_{n}$ whenever $n \geqslant n_{0}$.

The system of intervals

$$
\begin{array}{r}
\mathscr{J}=\left\{\left[x, x+\alpha_{n}\right]: n \geqslant n_{0}, 0 \leqslant x \leqslant 1-\beta_{n} \text { and } f(y)-f(x)>\sigma(y-x)\right. \\
\text { for every } \left.y \in\left[x+\alpha_{n}, x+\beta_{n}\right]\right\}
\end{array}
$$

is a Vitali cover of $E^{+}$. Therefore, by Vitali's theorem, there is a disjoint sequence of intervals $I_{k}=\left[x_{k}, x_{k}+\alpha_{n_{k}}\right] \in \mathscr{J}(k=1,2, \ldots)$ such that $\lambda\left(E^{+}\right) \leqslant \sum_{k=1}^{\infty}\left|I_{k}\right|$.

We show that the rectangles

$$
T_{k}=\left[x_{k}, x_{k}+\beta_{n_{k}}\right] \times\left[f\left(x_{k}\right), f\left(x_{k}\right)+\sigma\left(\alpha_{n_{k}}\right)\right] \quad(k=1,2, \ldots)
$$

are pairwise disjoint. Indeed, let $i \neq j$ be arbitrary. Then $I_{i} \cap I_{j}=\varnothing$; we can suppose $x_{i}+\alpha_{n_{i}}<x_{j}$. If $x_{i}+\beta_{n_{i}}<x_{j}$ then $T_{i} \cap T_{j}=\varnothing$ is obvious so that we can assume

$$
x_{i}+\alpha_{n_{i}}<x_{j} \leqslant x_{i}+\beta_{n_{i}} .
$$

Therefore, by $I_{i} \in \mathscr{J}$, we have $f\left(x_{j}\right)>f\left(x_{i}\right)+\sigma\left(x_{j}-x_{i}\right)$ and hence, for every $(x, y) \in T_{j}, y \geqslant f\left(x_{j}\right)>f\left(x_{i}\right)+\sigma\left(x_{j}-x_{i}\right) \geqslant f\left(x_{i}\right)+\sigma\left(\alpha_{n_{i}}\right)$, proving $T_{i} \cap T_{j}=\varnothing$. Obviously, $T_{k} \subset[0,1] \times[-K, K+\sigma(1)]$ for every $k=1,2, \ldots$ and hence

$$
\sum_{k=1}^{\infty} \beta_{n_{k}} \cdot \sigma\left(\alpha_{n_{k}}\right)=\sum_{k=1}^{\infty} \lambda_{2}\left(T_{k}\right)=\lambda_{2}\left(\bigcup_{k=1}^{\infty} T_{k}\right) \leqslant 2 K+\sigma(1) .
$$

Since $n_{k} \geqslant n_{0}$ for every $k$, this implies

$$
\lambda\left(E^{+}\right) \leqslant \sum_{k=1}^{\infty}\left|I_{k}\right|=\sum_{k=1}^{\infty} \alpha_{n_{k}}<\sum_{k=1}^{\infty} \varepsilon \sigma\left(\alpha_{n_{k}}\right) \beta_{n_{k}} \leqslant \varepsilon(2 K+\sigma(1)) .
$$

This proves $\lambda\left(E^{+}\right)=0$. A similar argument applies for $\lambda\left(E^{-}\right)=0$.

THEOREM 3. For every $\delta>0$ and every $f \in C[0,1]$ there is an absolutely continuous function $g$ such that $\{x: f(x)=g(x)\}$ is not bilaterally strongly $x^{1+\delta}$-porous.

Proof. We define $\beta_{n}=(n !)^{-1-\delta}$ and $\alpha_{n}=\beta_{n+1}, n=1,2, \ldots$ Let $\sigma(x)=n^{-1-\delta / 2}$ if $x \in\left(\alpha_{n}, \beta_{n}\right], n=1,2, \ldots$ Then

$$
\sigma\left(\alpha_{n}\right) \beta_{n} / \alpha_{n}=(n+1)^{-1-\delta / 2}(n+1)^{1+\delta} \rightarrow \infty,
$$

and hence by Theorem 2 for every $f \in C[0,1]$ there is an $x_{0} \in[0,1)$ and an $(\alpha, \beta)$-sequence $\left\{x_{n}\right\} \rightarrow x$ such that $x_{n}<1$ and

$$
\left|f\left(x_{n}\right)-f\left(x_{0}\right)\right| \leqslant \sigma\left(x_{n}-x_{0}\right) \leqslant \sigma\left(\beta_{n}\right)=n^{-1-\delta / 2} \text { for } n \geqslant n_{0} .
$$


Let $g$ be continuous on $[0,1]$, linear on the intervals $\left[0, x_{0}\right],\left[x_{n_{0}}, 1\right],\left[x_{n+1}, x_{n}\right]$, $n \geqslant n_{0}$, and agree with $f$ at the points $x_{n}, n \geqslant n_{0}$. Since $\sum_{n=1}^{\infty} n^{-1-\delta / 2}<\infty$, it is easy to see that $g$ is absolutely continuous on $[0,1]$. We show that $H=\{x: f(x)=g(x)\}$ is not bilaterally strongly $x^{1+\delta}$-porous at $x_{0}$. Indeed, for every interval $J$ with $J \subset\left(x_{0}, 1\right) \backslash H$ there is an $n$ such that $J \subset\left(x_{n+1}, x_{n}\right) \subset\left(x_{0}+\alpha_{n+1}, x_{0}+\beta_{n}\right)$. Therefore,

$$
\frac{\operatorname{dist}\left(x_{0}, J\right)}{|J|^{1+\delta}} \geqslant \frac{[(n+2) !]^{-1-\delta}}{(n !)^{-(1+\delta)^{2}}}=\left[\frac{(n !)^{\delta}}{(n+1)(n+2)}\right]^{1+\delta} \rightarrow \infty \quad \text { as } n \rightarrow \infty .
$$

\section{REFERENCES}

[H] J. Haussermann, Generalized porosity characteristics of a residual set of continuous functions, $\mathrm{Ph} . \mathrm{D}$. dissertation, University of California, Santa Barbara, 1984.

[T] B. S. Thomson, On the level set structure of a continuous function (to appear).

Department of Mathematics, St. Olaf College, Northfield, Minnesota 55057

Department of analysis, Eotvos Loránd University, Budapest, Múzeum krt. 6-8, Hungary H - 1088 